\title{
Enantio- and Structure-Selective Diels-Alder Reactions of Unsymmetrical Quinones Catalyzed by a Chiral Oxazaborolidinium Cation. Predictive Selection Rules.
}

\author{
Do Hyun Ryu, Gang Zhou and E. J. Corey* \\ Department of Chemistry and Chemical Biology \\ Harvard University, Cambridge, Massachusetts, 02138, USA
}

\section{$\underline{\text { Supplementary Material }}$}

Materials and Methods. Unless stated otherwise, reactions were performed in flame-dried glassware under a positive pressure of nitrogen using freshly distilled solvents. Thin-layer chromatography (TLC) was performed using E. Merck silica gel $60 \mathrm{~F}_{254}$ precoated plates $(0.25$ $\mathrm{mm}$ ). Flash chromatography was performed using Baker silica gel (40 $\mu \mathrm{m}$ particle size). NMR spectra were recorded on Varian Innova-500, or Mercury-400 instruments and calibrated using residual undeuterated solvent as an internal reference. IR spectra were recorded on Avatar 360 FT-IR spectrometer. Low-resolution mass (CI) spectra were obtained by using a Platform II mass spectrometer. High-resolution mass spectral analyses were performed at the Harvard University Mass Spectrometry Center. Analytical high performance liquid chromatography (HPLC) was performed on Isco 2350 Series or Waters 626 HPLC using the indicated chiral column. Gas chromatography (GC) analyses were performed on Hewlett-Packard 6850 Series GC System equipped with flame ionization detector using a J \& W Scientific Cyclosil-B column (30 m x $0.25 \mathrm{~mm}$ ). Commercial grade reagents and solvents were used without further purification except as indicated below. Dichloromethane was distilled from calcium hydride. Toluene was distilled from sodium. 2-Methylnaphthalene-1, 4-dione (Aldrich), naphthalene-1, 4-dione (Aldrich), 2bromo-6-methyl-1,4-benzoquinone (Sigma-Aldrich), 1,4-benzoquinone (Aldrich) and 2-t-butyl1,4-benzoquinone were used after recrystallization with a mixture of ethyl acetate and hexanes. 2-Triisopropylsilyloxy-1,3-butadiene, ${ }^{1} \quad 2$, 3, 5-trimethyl-1,4-benzoquinone, ${ }^{2}$ 1-bromo-2, 4-

\footnotetext{
${ }^{1}$ Corey, E. J.; Guzman-Perez, A.; Loh, T.-P. J. Am. Chem. Soc. 1994, 116, 3611.

${ }^{2}$ Ito, S.; Aihara, K.; Matsumoto, M. Tetrahedron Lett. 1983, 24, 5249.
} 
dimethyl-1, 4-benzoquinone, ${ }^{3}$ 1-methoxy-2-methyl-1, 4-benzoquinone, ${ }^{4}$ 2, 3-dimethyl-1, 4benzoquinone, ${ }^{5}$ 2-methoxy-6-methyl-1, 4-benzoquinone ${ }^{6}$ and 3-iodo-2, 5-dimethyl-1, 4benzoquinone ${ }^{7}$ were prepared according to literature procedures.

General Procedure for the Preparation and Use of Chiral Diels-Alder Catalysts. A 100-mL, two-necked, round-bottomed flask equipped with a stir bar, a glass stopper and a 50-mL pressure-equalizing addition funnel (containing a cotton plug and ca. $10 \mathrm{~g}$ of 4 molecular sieves, ${ }^{8}$ and functioning as a Soxhlet extractor) fitted on top with a reflux condenser and a nitrogen inlet adaptor was charged with (S)-(-)- $\alpha, \alpha$-diphenyl-2-pyrrolidinemethanol (82.0 mg, $0.324 \mathrm{mmol}$, from Lancaster), tri-o-tolylboroxine ${ }^{9}(38.0 \mathrm{mg}, 0.107 \mathrm{mmol})$ and $25 \mathrm{~mL}$ of toluene. The resulting solution was heated to reflux (bath temperature $\sim 145^{\circ} \mathrm{C}$ ). After $3 \mathrm{~h}$, the reaction mixture was cooled to $c a .60{ }^{\circ} \mathrm{C}$ and the addition funnel and condenser were quickly replaced with a short-path distillation head. The mixture was concentrated by distillation (air-cooling) to a volume of $c a .5 \mathrm{~mL}$. This distillation protocol was repeated three times by re-charging with $3 \mathrm{x}$ $5 \mathrm{~mL}$ of toluene. The solution was then allowed to cool to room temperature and the distillation head was quickly replaced with a vacuum adaptor. Concentration in vacuo (ca. $0.1 \mathrm{mmHg}, 1 \mathrm{~h}$ ) afforded the corresponding oxazaborolidine as clear oil, which can then be dissolved in $\mathrm{CH}_{2} \mathrm{Cl}_{2}$ and used in two Diels-Alder experiments.

To an aliquot of the oxazaborolidine precursor $(0.160 \mathrm{mmol}$, theoretical $)$ in $1.0 \mathrm{~mL} \mathrm{of} \mathrm{CH}_{2} \mathrm{Cl}_{2}$ at $-25^{\circ} \mathrm{C}$ was added trifluoromethanesulfonimide $\left(0.20 \mathrm{M}\right.$ solution in $\mathrm{CH}_{2} \mathrm{Cl}_{2}$, freshly prepared, $667 \mu \mathrm{L}, 0.133 \mathrm{mmol}$ ) dropwise. After $10 \mathrm{~min}$ at $-25^{\circ} \mathrm{C}$, a colorless homogeneous catalyst solution was ready for use in the Diels-Alder reactions.

\footnotetext{
3 a) Smith, L. I.; Wiley, P.F. J. Am. Chem. Soc. 1946, 68, 887. b) Smith, L. I.; Wiley, P.F. J. Am. Chem. Soc. 1946, 68, 894.

${ }^{4}$ Knolker, H-J.; Frohner, W.; Reddy, K. R. Synthesis 2002, 4, 557.

${ }^{5}$ Fischt, S.; Mulbaier, M.; Giannis, A. Tetrahedron 2001, 57, 4863.

${ }^{6}$ Stevens, R. V.; Angle, S. R.; Kloc, K.; Mak, K. F.; Trueblood, K. L.; Liu, Y.-X. J. Org. Chem. 1986, $51,4347$.

${ }^{7}$ Cressman, H. W. J.; Thirtle, J. R. J. Org. Chem. 1966, 31, 1279.

${ }^{8}$ Molecular sieves (pellets) were dried in vacuo at ca. $200{ }^{\circ} \mathrm{C}$ with a gas burner for 10 min prior to use.

${ }^{9}$ Corey, E. J.; Shibata, T.; Lee, T. W. J. Am. Chem. Soc. 2002, 124, 3803.
} 
To a catalyst solution in $\mathrm{CH}_{2} \mathrm{Cl}_{2}(1.0 \mathrm{~mL})$ were successively added a solution of 1 , 4benzoquinone $(0.665 \mathrm{mmol})$ in $\mathrm{CH}_{2} \mathrm{Cl}_{2}(0.7 \sim 1.0 \mathrm{~mL})$ and 2-triisopropylsilyloxy-1,3-butadiene $(194 \mu \mathrm{L}, 166 \mathrm{mg}, 0.732 \mathrm{mmol})$ at $-78^{\circ} \mathrm{C}$. The reaction mixture was stirred at the temperature indicated in Table 1-3 and monitored by ${ }^{1} \mathrm{H}$ NMR or TLC. When it was judged to be complete, dichloromethane was removed by rotary evaporation and water $(5 \mathrm{~mL})$ and hexanes $(3 \mathrm{~mL})$ were added. The aqueous layer was extracted with hexanes $(4 \mathrm{x} 5 \mathrm{~mL}){ }^{10}$ The combined extract was dried over anhydrous $\mathrm{Na}_{2} \mathrm{SO}_{4}$ and concentrated to afford the corresponding adduct which was pure enough for the most purposes. Further purification was performed by silica gel chromatography (Table 1), low temperature $\left(-78^{\circ} \mathrm{C}\right)$ silica gel chromatography or recrystallization (Table 2,3 ). ${ }^{11}$

The corresponding racemic products for the determination of enantioselectivity were prepared by using ethylaluminum dichloride $(20 \mathrm{~mol} \%)$ or anhydrous $\mathrm{LiClO}_{4}{ }^{12}$ as a Lewis acid catalyst or thermal reaction.

\section{Determination of Regioselectivity, Enantioselectivity and Absolute Configuration of the}

\section{Diels-Alder Adducts Reported in Tables 1-3.}

Table 1, entry 1: Regioselectivity and enantioselectivity were determined by HPLC analysis using a Regis $(S, S)$-Whelk-O1 column $(0.2 \% i$-PrOH in hexanes for elution; $1 \mathrm{~mL} / \mathrm{min} ; \lambda 254$ $\mathrm{nm}$ ); retention times: $13.1 \mathrm{~min}$ (major), $15.5 \mathrm{~min}$ (minor), $18.6 \mathrm{~min}$ (regioisomer). The absolute configuration was determined by comparison with an authentic sample prepared from (-)-(4aR, 8aS)-3-iodo -2, 4a -dimethyl-6 -(triisopropylsilyl)oxy - 4a, 5, 8, 8a - tetrahydro -1, 4naphthoquinone (substitution of iodine with methyl group ${ }^{13}$ ) and HPLC analysis.

\footnotetext{
10 After hexanes extraction, the aqueous solution was treated with aqueous $2 \mathrm{~N} \mathrm{NaOH}$ solution and extracted with dichloromethane. The combined extract was dried over $\mathrm{Na}_{2} \mathrm{SO}_{4}$, concentrated and purified by column chromatography (elution with EtOAc) to afford pure ligand (95-99\% recovery).

${ }^{11}$ Quinone adducts in Table 2 and 3 are unstable under silica gel chromatography at ambient temperature.

${ }^{12}$ Grieco, P. A.; Nunes, J. J.; Gaul, M. D. J. Am. Chem. Soc. 1990, 112, 4595.

${ }^{13}$ The solution of iodoquinone adduct (Table 1, entry 3) in THF was treated with dimethylzinc (2.0 eq.) in the presence of catalytic amount of dichlorobis(triphenylphosphine)palladium for $3 \mathrm{~h}$ at $20{ }^{\circ} \mathrm{C}$ to provide the corresponding methyl quinone adduct ( Table 1 , entry $1,56 \%$ yield).
} 
Table 1, entry 2: Regioselectivity and enantioselectivity were determined by HPLC analysis using a Daicel Chiralcel OD-H column $(0.2 \% i$-PrOH in hexanes for elution; $1 \mathrm{~mL} / \mathrm{min} ; \lambda 254$ $\mathrm{nm}$ ); retention times: $12.2 \mathrm{~min}$ (major), $14.6 \mathrm{~min}$ (regioisomer), $15.6 \mathrm{~min}$ (regioisomer), 16.3 $\min ($ minor). The absolute configuration was determined by X-ray crystallographic analysis.

Table 1, entry 3: Enantioselectivity was determined by subsequent desilylation with $10 \% \mathrm{HF}$, reduction with $n \mathrm{Bu}_{3} \mathrm{SnH}$ to the ketone compound and $\mathrm{GC}$ analysis $\left(120^{\circ} \mathrm{C}, 25 \mathrm{psi}\right)$; retention times: 169.4 (major), 172.5 min (minor). The absolute configuration was assigned by X-ray crystallographic analysis of $(4 a R, 8 a S)-3$-iodo -2, 4a -dimethyl -4a, 7, 8, 8a -tetrahydro- $5 H$ -naphthalene -1, 4, 6-trione; Ryu, D. H.; Corey, E. J. J. Am. Chem. Soc. 2003, 125, 6388.

Table 1, entry 4: Regioselectivity and enantioselectivity were determined by HPLC analysis using a Daicel Chiralcel OD-H column $(0.1 \% i$-PrOH in hexanes for elution; $1 \mathrm{~mL} / \mathrm{min} ; \lambda 254$ $\mathrm{nm}$ ); retention times: $34.5 \mathrm{~min}$ (major), $42.6 \mathrm{~min}$ (minor), $38.7 \mathrm{~min}$ (regioisomer), 40.1 min(regioisomer). The absolute configuration was determined by analogy with (-)-(4aR, $8 a S)-2-$ bromo-4a, 5, 8, 8a-tetrahydro-6-(triisopropylsilyl)oxy-3, 4a-dimethyl-naphthalene-1, 4-dione.

Table 1, entry 5: Enantioselectivity was determined by HPLC analysis using a Daicel Chiralcel OD-H column $(0.4 \% i$-PrOH in hexanes for elution; $1 \mathrm{~mL} / \mathrm{min} ; \lambda 254 \mathrm{~nm})$; retention times: 9.7 min (major), 11.8 min (minor). The absolute configuration was determined by selective reduction with $\mathrm{NaBH}_{4}, p$-bromobenzoylation, desilylation and X-ray crystallographic analysis of $(4 a S, 9 R, 9 a R)-1,2,3,4,4 a, 9,9 a, 10$ - octahydro- 9a- methyl- 2, 10- dioxoanthracen-9-yl-4bromobenzoate (vide infra).

Table 2, entry 1: Enantioselectivity was determined by reduction with $\mathrm{NaBH}_{4}$ to the corresponding diol, conversion to the $(R)$-MTPA ester derivative and ${ }^{1} \mathrm{H}$ NMR integration (500 $\mathrm{MHz}_{\mathrm{CDCl}}$ ): $\delta 6.64(\mathrm{dd}, 1 \mathrm{H}, J=5.0 \mathrm{~Hz}$, major), 6.51 (d, 1H, $J=4.5 \mathrm{~Hz}$, minor). The absolute configuration was determined by analogy with (+)-(4aR, 8aS)-4a, 5, 8, 8a-tetrahydro-6(triisopropylsilyl)oxynaphthalene-1, 4-dione and (+)-(4aS, 9aR)-1, 4, 4a, 9a-tetrahydro-2(triisopropylsilyl)oxy-9a-methylanthracene-9, 10-dione. 
Table 2, entry 2: Enantioselectivity was determined by desilylation with $10 \%$ HF to the corresponding ketone and $\mathrm{GC}$ analysis $\left(155^{\circ} \mathrm{C}, 25 \mathrm{psi}\right)$; retention times: 58.4 (major), $59.7 \mathrm{~min}$ (minor). The absolute configuration was determined by analogy with (+)-(4aR, $8 a S)-2,3,6-$ trimethyl-4a, 5, 8, 8a-tetrahydro-1, 4-naphthoquinone; Ryu, D. H.; Corey, E. J. J. Am. Chem. Soc. 2003, 125, 6388 .

Table 2, entry 3: Regioselectivity was determined by ${ }^{1} \mathrm{H}$ NMR analysis of the crude mixture: $\delta 3.25(\mathrm{dd}, 1 \mathrm{H}, J=15.0,8.0 \mathrm{~Hz}$, major), $3.20(\mathrm{dd}, 1 \mathrm{H}, J=15.0,8.0 \mathrm{~Hz}$, minor). Enantioselectivity was determined by selective Luche reduction with $\mathrm{NaBH}_{4}$ to the corresponding alcohol, conversion to the $(R)$-MTPA ester derivative and ${ }^{1} \mathrm{H}$ NMR integration $\left(500 \mathrm{MHz}, \mathrm{CDCl}_{3}\right): \delta 6.26(\mathrm{dd}, 1 \mathrm{H}, J=5.5,3.2 \mathrm{~Hz}$, major), $6.13(\mathrm{~d}, 1 \mathrm{H}, J=4.0 \mathrm{~Hz}$, minor). The absolute configuration was determined by analogy with (+)-(4aR, 8aS)- 2, 3-Dimethyl-6(triisopropylsilyl)oxy - 4a, 5, 8, 8a -tetrahydro -1, 4-naphthoquinone; Ryu, D. H.; Corey, E. J. J. Am. Chem. Soc. 2003, 125, 6388.

Table 2, entry 4: Enantioselectivity was determined by desilylation to the corresponding ketone and HPLC analysis using a Daicel Chiralcel OJ column (50\% $i$-PrOH in hexanes for elution; 0.5 $\mathrm{mL} / \mathrm{min} ; \lambda 254 \mathrm{~nm}$ ); retention times: $39.7 \mathrm{~min}$ (minor), $44.1 \mathrm{~min}$ (major). The absolute configuration was assigned by comparison with an authentic sample prepared from (-)(4aS, 8aR) -4a, 5, 8, 8a-tetrahydro-2-bromo-7-(triisopropylsilyl)oxy-8a-methylnaphthalene-1, 4dione (substitution of bromine with methoxy group, ${ }^{14}$ desilylation) and HPLC analysis.

Table 2, entry 5: Enantioselectivity was determined by substitution of bromine with methoxy group, desilylation and HPLC analysis using a Daicel Chiralcel OJ column $(50 \% i$-PrOH in hexanes for elution; $0.5 \mathrm{~mL} / \mathrm{min} ; \lambda 254 \mathrm{~nm}$ ); retention times: $39.7 \mathrm{~min}$ (minor), $44.1 \mathrm{~min}$ (major). The absolute configuration was determined by desilylation and X-ray crystallographic

\footnotetext{
${ }^{14}$ The bromoquinone adduct (Table 2, entry 5) was stirred for $24 \mathrm{hr}$ at $20{ }^{\circ} \mathrm{C}$ under basic methanol solution $(1 \mathrm{~N}$ $\mathrm{NaOH}: \mathrm{MeOH}=1: 50$ ) to give the corresponding methoxy quinone adduct (Table 2, entry 4) and its trans isomer (= $1: 1,85 \%$ yield).
} 
analysis of $(4 a R, 8 a S)$-3-bromo-4a, 5, 8, 8a-tetrahydro-4a-methylnaphthalene-1, 4, 6(7H)-trione (vide infra).

Table 3, entry 1: Enantioselectivity was determined by reduction with $\mathrm{NaBH}_{4}$ to the corresponding diol, then acid-catalyzed cyclization to cyclic ketal monoalcohol, conversion to the (R)-MTPA ester derivative and ${ }^{1} \mathrm{H}$ NMR integration $\left(500 \mathrm{MHz}, \mathrm{CDCl}_{3}\right): \delta 6.03(\mathrm{dd}, 0.5 \mathrm{H}, J=$ $2.0 \mathrm{~Hz}$, minor), 5.97 (dd, $0.5 \mathrm{H}, J=2.0 \mathrm{~Hz}$, major); ${ }^{19} \mathrm{~F} \mathrm{NMR} \mathrm{integration}\left(376.2 \mathrm{MHz}, \mathrm{CDCl}_{3}\right.$ ): $\delta-71.79$ ( $\mathrm{s}, \mathrm{CF}_{3}$, major), $-71.89\left(\mathrm{~s}, \mathrm{CF}_{3}\right.$, minor). The absolute configuration was assigned by selective Luche reduction, acid-catalyzed ketal formation, $p$-bromobenzoylation, desilylation with $10 \% \mathrm{HF}$ (in $\mathrm{CH}_{3} \mathrm{CN}$ ) and X-ray crystallographic analysis of $(1 R, 4 S, 4 a R, 8 a S)-1,4,4 \mathrm{a}, 5,6$, 7, 8, 8a-octahydro-1-hydroxy-6-oxonaphthalen-4-yl-4-bromobenzoate (vide infra).

Table 3, entry 2: Regioselectivity and enantioselectivity were determined by reduction with $\mathrm{NaBH}_{4}$ to the corresponding alcohol, conversion to the ( $R$ )-MTPA ester derivative and ${ }^{1} \mathrm{H}$ NMR integration $\left(500 \mathrm{MHz}, \mathrm{CDCl}_{3}\right): \delta 6.65(\mathrm{t}, 1 \mathrm{H}, J=2.0 \mathrm{~Hz}$, minor), $6.63(\mathrm{t}, 1 \mathrm{H}, J=2.0 \mathrm{~Hz}$, regioisomer), $6.58(\mathrm{t}, 1 \mathrm{H}, J=2.0 \mathrm{~Hz}$, regioisomer), $6.55(\mathrm{t}, 1 \mathrm{H}, J=2.0 \mathrm{~Hz}$, major). The absolute configuration was determined by analogy with (+)-(4aS, 8aR)-4a, 5, 8, 8a-tetrahydro-2methoxy-7-(triisopropylsilyl)oxy-naphthalene-1,4-dione.

Table 3, entry 3: Regioselectivity was determined by ${ }^{1} \mathrm{H}$ NMR analysis of the crude mixture: $\delta 3.04(\mathrm{dd}, 1 \mathrm{H}, J=12.4,6.4 \mathrm{~Hz}$, major), $3.08(\mathrm{dd}, 1 \mathrm{H}, J=11.6,5.6 \mathrm{~Hz}$, minor). Enantioselectivity was determined by reduction with $\mathrm{NaBH}_{4}$ to the corresponding alcohol, conversion to the (R)-MTPA ester derivative and ${ }^{1} \mathrm{H}$ NMR integration (500 MHz, $\mathrm{CDCl}_{3}$ ): $\delta 4.71$ (d, $1 \mathrm{H}, J=5.2 \mathrm{~Hz}$, major), $4.63(\mathrm{~m}, 1 \mathrm{H}$, minor). The absolute configuration was determined by analogy with (+)-(4aS,8aR)-4a, 5, 8, 8a-tetrahydro-2-methoxy-7-(triisopropylsilyl)oxynaphthalene-1,4-dione.

Table 3, entry 4: Enantioselectivity was determined by HPLC analysis using a Daicel Chiralcel OD-H column ( $0.4 \% i$-PrOH in hexanes for elution; $1 \mathrm{~mL} / \mathrm{min} ; \lambda 254 \mathrm{~nm})$; retention times: 9.7 min (major), 11.8 min (minor). The absolute configuration was determined by selective reduction with $\mathrm{NaBH}_{4}, p$-bromobenzoylation, desilylation and X-ray crystallographic analysis of 
$(4 a S, 9 R, 9 a R)-1,2,3,4,4 a, 9,9 a, 10$ - octahydro- 9a- methyl- 2, 10- dioxoanthracen-9-yl 4bromobenzoate (vide infra).

\section{Experimental Procedure and Physical Data of the Diels-Alder Products: ${ }^{15}$}

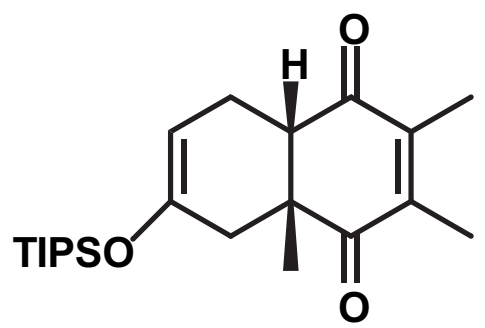

(4aR, 8aS)-4a, 5, 8, 8a-Tetrahydro-6-(triisopropylsilyl)oxy-2, 3, 4a-trimethylnaphthalene-1,

4-dione (Table 1, entry 1). Purification by column chromatography (elution with pentane) afforded $246 \mathrm{mg}(98 \%)$ of product as a colorless oil: ${ }^{1} \mathrm{H} \mathrm{NMR}\left(400 \mathrm{MHz}, \mathrm{CDCl}_{3}\right) \delta 4.80(\mathrm{t}, 1 \mathrm{H}$, $J=3.6 \mathrm{~Hz}), 2.74(\mathrm{dd}, 1 \mathrm{H}, J=6.0,5.2 \mathrm{~Hz}), 2.56-2.68(\mathrm{~m}, 1 \mathrm{H}), 2.52(\mathrm{dd}, 1 \mathrm{H}, J=17.2,1.6 \mathrm{~Hz})$, 2.12-2.22 (m, 1H), 1.99 (s, 3H), $1.96(\mathrm{~s}, 3 \mathrm{H}), 1.79$ (d, 1H, J=17.2 Hz), 1.32 (s, 3H), 1.01-1.12 $(\mathrm{m}, 3 \mathrm{H}), 1.03(\mathrm{~d}, 18 \mathrm{H}, J=6.0 \mathrm{~Hz}) ;{ }^{13} \mathrm{C} \mathrm{NMR}\left(100 \mathrm{MHz}, \mathrm{CDCl}_{3}\right) \delta$ 201.9, 199.6, 147.8, 143.6, 142.6, 99.9, 51.8, 48.8, 37.1, 23.6, 23.1, 18.2 (x6), 13.4, 13.1, 12.8 (x3); FTIR (neat) 2924, 1674, 1489, 1204; LRMS (CI) calcd for $\left[\mathrm{C}_{22} \mathrm{H}_{37} \mathrm{O}_{3} \mathrm{Si}\right]\left([\mathrm{MH}]^{+}\right)$: 377; found $377 ;[\alpha]_{\mathrm{D}}^{23}-33.8(c 1.1$, $\mathrm{CHCl}_{3}, 99 \%$ ee).

\footnotetext{
${ }^{15}$ The physical and spectral data of two Diels-Alder adducts are known. For $(4 a R, 8 a S)$ - 3 -iodo $-2,4 a-$ dimethyl -6 -(triisopropyl silyl)oxy - 4a, 5, 8, 8a - tetrahydro -1,4- naphthoquinone and (4aR, 8aS)- 2, 3 - dimethyl - 6 (triisopropyl silyl)oxy - 4a, 5, 8, 8a - tetrahydro -1,4 naphthoquinone, see Ryu, D. H.; Corey, E. J. J. Am. Chem. Soc. 2003, 125, 6388.
} 


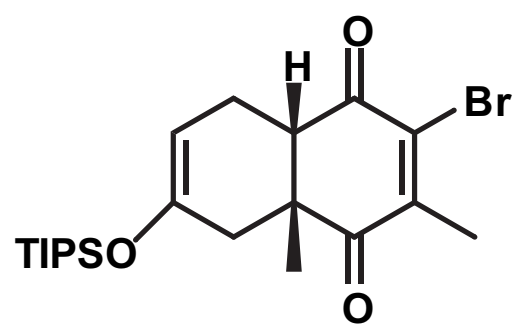

(4aR, $8 a S)$-2-Bromo-4a, 5, 8, 8a-tetrahydro-6-(triisopropylsilyl)oxy-3, 4a-dimethylnaphthalene-1, 4-dione (Table 1, entry 2). Purification by column chromatography (gradient elution with $1 \% \mathrm{Et}_{2} \mathrm{O}$-pentane) afforded $282 \mathrm{mg}(96 \%)$ of product as a pale yellow solid: $\mathrm{mp}$ 68.0-70.0 ${ }^{\circ} \mathrm{C}:{ }^{1} \mathrm{H}$ NMR $\left(500 \mathrm{MHz}, \mathrm{CDCl}_{3}\right) \delta 4.80(\mathrm{t}, 1 \mathrm{H}, J=4.0 \mathrm{~Hz}), 2.87(\mathrm{dd}, 1 \mathrm{H}, J=6.0,6.0$ $\mathrm{Hz}), 2.60-2.64(\mathrm{~m}, 1 \mathrm{H}), 2.54-2.58(\mathrm{dd}, 1 \mathrm{H}, J=17.5,1.5 \mathrm{~Hz}), 2.23-2.67(\mathrm{~m}, 1 \mathrm{H}), 2.22(\mathrm{~s}, 3 \mathrm{H})$, 1.85(d, $1 \mathrm{H}, J=17.5 \mathrm{~Hz}), 1.36(\mathrm{~s}, 3 \mathrm{H}), 1.01-1.15(\mathrm{~m}, 3 \mathrm{H}), 1.04(\mathrm{~d}, 18 \mathrm{H}, J=6.0 \mathrm{~Hz}) ;{ }^{13} \mathrm{C} \mathrm{NMR}$ $\left(125 \mathrm{MHz}, \mathrm{CDCl}_{3}\right) \delta 197.9,191.7,147.9,147.7,138.7,99.4,51.4,49.0,36.7,23.6,23.57,18.4$, 18.2 (x6), 12.8 (x3); FTIR (neat) 2941, 2864, 1688, 1462, 1202; LRMS (CI) calcd for $\left[\mathrm{C}_{21} \mathrm{H}_{33} \mathrm{BrO}_{3} \mathrm{Si}\right]\left([\mathrm{M}]^{-}\right): 440,442$; found 440, 442; $[\alpha]_{\mathrm{D}}^{23}-2.0\left(c 0.8, \mathrm{CHCl}_{3}, 97 \%\right.$ ee $)$.

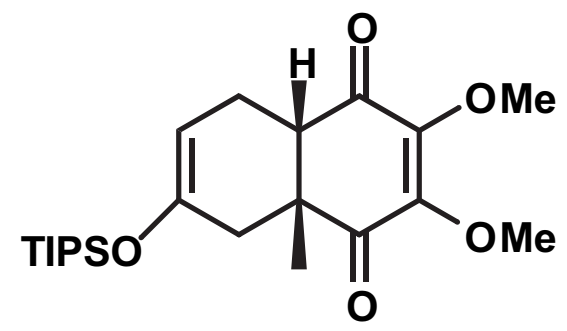

(4aR, 8aS)-4a, 5, 8, 8a-Tetrahydro-2, 3-dimethoxy-6- （triisopropylsilyl)oxy-4a-methylnaphthalene-1, 4-dione (Table 1, entry 4). Purification by column chromatography (gradient elution with hexanes and 5\% $\mathrm{Et}_{2} \mathrm{O}$-pentane) afforded $230 \mathrm{mg}(85 \%)$ of product as a colorless oil: ${ }^{1} \mathrm{H}$ NMR $\left(500 \mathrm{MHz}, \mathrm{CDCl}_{3}\right) \delta 4.79(\mathrm{~m}, 1 \mathrm{H}), 3.96(\mathrm{~s}, 6 \mathrm{H}), 2.68(\mathrm{~m}, 1 \mathrm{H}), 2.63-2.69(\mathrm{~m}, 1 \mathrm{H}), 2.53-$ $2.58(\mathrm{dd}, 1 \mathrm{H}, J=17.2,1.5 \mathrm{~Hz}), 2.19-2.23(\mathrm{~m}, 1 \mathrm{H}), 1.85(\mathrm{~d}, 1 \mathrm{H}, J=17.2 \mathrm{~Hz}), 1.35$ (s, 3H), 1.01$1.24(\mathrm{~m}, 3 \mathrm{H}), 1.04(\mathrm{~d}, 18 \mathrm{H}, J=6.0 \mathrm{~Hz}) ;{ }^{13} \mathrm{C} \mathrm{NMR}\left(100 \mathrm{MHz}, \mathrm{CDCl}_{3}\right) \delta 197.8,195.4,147.8$, 147.6, 147.0, 99.4, 60.84, 60.78, 50.9, 48.0, 37.0, 23.6, 22.9, 18.2 (x6), 12.8 (x3); FTIR (neat) 
2942, 2865, 1677, 1599, 1281, 1208; HRMS (CI) calcd for $\left[\mathrm{C}_{22} \mathrm{H}_{37} \mathrm{O}_{5} \mathrm{Si}\right]\left([\mathrm{MH}]^{+}\right): 409.2410$; found 409.2420; $[\alpha]_{\mathrm{D}}^{23}-6.6\left(c 1.5, \mathrm{CHCl}_{3}, 95 \%\right.$ ee $)$.

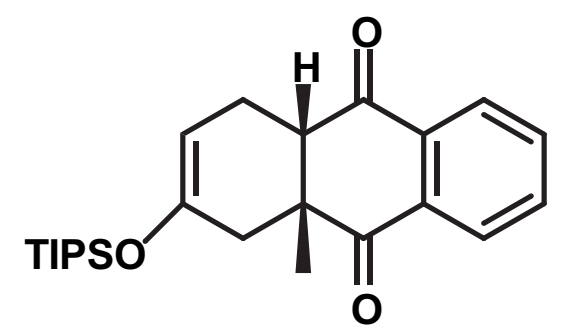

(4aS,9aR)-1,4,4a,9a-Tetrahydro-2-(triisopropylsilyl)oxy-9a-methylanthracene-9,10-dione

(Table 1, entry 5). Purification by column chromatography (5\% EtOAc-hexanes) afforded 255 mg $(96 \%)$ of product as a white solid: $\mathrm{mp} 45.0-46.0{ }^{\circ} \mathrm{C}:{ }^{1} \mathrm{H} \mathrm{NMR}\left(500 \mathrm{MHz}, \mathrm{CDCl}_{3}\right) \delta$ 8.05-8.06 (m, 1H), 8.00-8.02 (m, 1H), 7.69-7.74 (m, 2H), 4.81-4.83 (m, 1H), 2.94 (dd, 1H, J = 7.0, 5.5 Hz), $2.72(\mathrm{dd}, 1 \mathrm{H}, J=17.0,1.5 \mathrm{~Hz}), 2.50-2.56(\mathrm{~m}, 1 \mathrm{H}), 2.24-2.30(\mathrm{~m}, 1 \mathrm{H}), 1.90(\mathrm{dd}, 1 \mathrm{H}, J=17.0,1.5$ $\mathrm{Hz}), 1.38(\mathrm{~s}, 3 \mathrm{H}), 1.07-1.16(\mathrm{~m}, 3 \mathrm{H}), 1.04(\mathrm{~d}, 18 \mathrm{H}, J=6.5 \mathrm{~Hz}) ;{ }^{13} \mathrm{C} \mathrm{NMR}\left(100 \mathrm{MHz}, \mathrm{CDCl}_{3}\right)$ $\delta 199.1,198.0,147.9,134.2,134.0,133.3,132.8,127.2,126.4,99.3,52.6,48.8,36.4,23.8$ (x2), 17.8 (x6), 12.4 (x3); FTIR (neat) 2941, 2864, 1693, 1674, 1202; LRMS (CI) calcd for $\left[\mathrm{C}_{24} \mathrm{H}_{34} \mathrm{O}_{3} \mathrm{Si}\right]\left([\mathrm{M}]^{-}\right)$: 398; found 398; $[\alpha]_{\mathrm{D}}^{23}+23.6\left(c 1.0, \mathrm{CHCl}_{3}, 91 \%\right.$ ee $)$.

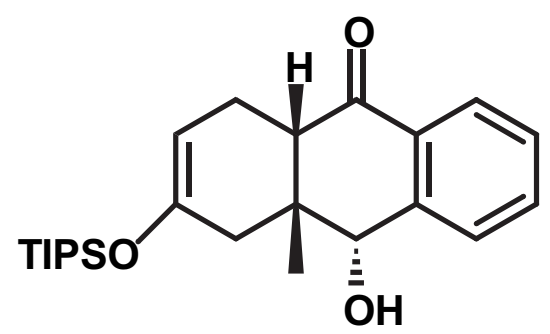

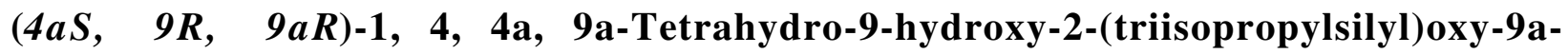
methylanthracen-10 $(9 \mathrm{H})$-one. To a stirred solution of the (triisopropylsilyl)oxy quinone adduct (99.6 mg, $0.25 \mathrm{mmol})$ in $\mathrm{MeOH}(3.5 \mathrm{~mL})$ was added sodium borohydride $(11.3 \mathrm{mg}, 0.29 \mathrm{mmol})$ at $0{ }^{\circ} \mathrm{C}$ and the reaction mixture was stirred for $30 \mathrm{~min}$ at $0{ }^{\circ} \mathrm{C}$. Water $(4 \mathrm{~mL})$ and EtOAc $(4 \mathrm{~mL})$ were added and the layers were separated. The aqueous layer was extracted with EtOAc (4 mLx3). The combined extract was dried over sodium sulfate and concentrated in vacuo to give a 
white solid product (99.1mg, 99\%): $\mathrm{mp} 108.0-109.0{ }^{\circ} \mathrm{C}$ (EtOAc-hexanes): ${ }^{1} \mathrm{H}$ NMR (500 MHz, $\left.\mathrm{CDCl}_{3}\right) \delta 7.98(\mathrm{dd}, 1 \mathrm{H}, J=7.5,1.0 \mathrm{~Hz}), 7.76(\mathrm{~d}, 1 \mathrm{H}, J=8.5 \mathrm{~Hz}), 7.58(\mathrm{ddd}, 1 \mathrm{H}, J=7.5,7.5,1.0$ $\mathrm{Hz}), 7.35$ (dd, 1H, $J=7.5,7.5 \mathrm{~Hz}), 4.84(\mathrm{~s}, 1 \mathrm{H}), 4.81-4.82(\mathrm{~m}, 1 \mathrm{H}), 3.02$ (dd, 1H, $J=17.0,5.5$ Hz), 2.92 (brs, 1H), 2.34 (d, 1H, $J=5.5 \mathrm{~Hz}), 2.21-2.27$ (m, 1H), 1.95 (d, 1H, $J=17.5 \mathrm{~Hz}), 1.76$ $(\mathrm{d}, 1 \mathrm{H}, J=17.5 \mathrm{~Hz}), 1.38(\mathrm{~s}, 3 \mathrm{H}), 0.99-1.08(\mathrm{~m}, 3 \mathrm{H}), 0.95(\mathrm{~d}, 9 \mathrm{H}, J=7.0 \mathrm{~Hz}), 0.94(\mathrm{~d}, 9 \mathrm{H}, J=$ $6.5 \mathrm{~Hz}) ;{ }^{13} \mathrm{C} \mathrm{NMR}\left(125 \mathrm{MHz}, \mathrm{CDCl}_{3}\right) \delta 196.9,148.2,143.8,134.0,130.3,127.6,126.8,126.4$, 100.7, 75.6, 50.0, 41.8, 32.0, 23.7, 20.2, 17.8 (x6), 12.4 (x3); FTIR (neat) 2940, 2864, 1665, 1206; LRMS (CI) calcd for $\left[\mathrm{C}_{24} \mathrm{H}_{33} \mathrm{O}_{3} \mathrm{Si}\right]\left([\mathrm{M}-\mathrm{H}]^{-}\right)$: 399; found 399; $[\alpha]_{\mathrm{D}}^{23}-67.5$ (c 1.0, $\mathrm{CHCl}_{3}$, $91 \%$ ee).

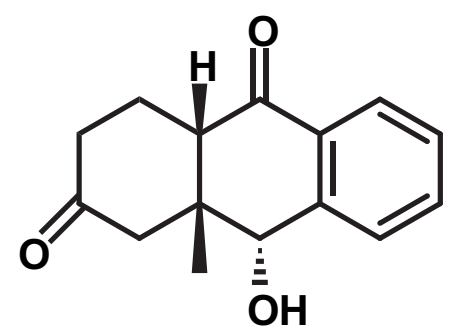

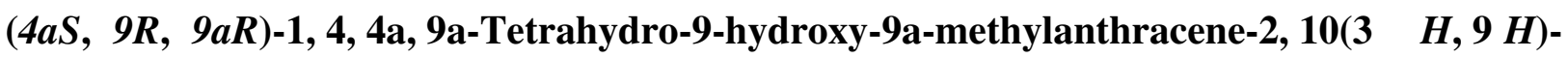
dione. Deprotection of TIPS group ${ }^{16}$ afforded $35 \mathrm{mg}$ (95\%) of ketone product as a colorless oil: ${ }^{1} \mathrm{H}$ NMR $\left(500 \mathrm{MHz}, \mathrm{CDCl}_{3}\right) \delta 8.10(\mathrm{~d}, 1 \mathrm{H}, J=7.5 \mathrm{~Hz}), 7.62-7.69(\mathrm{~m}, 2 \mathrm{H}), 7.45-7.49(\mathrm{~m}, 1 \mathrm{H})$, $4.82(\mathrm{~d}, 1 \mathrm{H}, J=4.5 \mathrm{~Hz}), 2.77-2.83(\mathrm{~m}, 1 \mathrm{H}), 2.65(\mathrm{t}, 1 \mathrm{H}, J=5.0 \mathrm{~Hz}), 2.32-2.42(\mathrm{~m}, 2 \mathrm{H}), 2.30(\mathrm{~d}$, $1 \mathrm{H}, J=15.0 \mathrm{~Hz}), 2.24(\mathrm{~d}, 1 \mathrm{H}, J=15.0 \mathrm{~Hz}), 2.04-2.12(\mathrm{~m}, 1 \mathrm{H}), 1.26(\mathrm{~s}, 3 \mathrm{H}) ;{ }^{13} \mathrm{C} \mathrm{NMR}(100$ $\left.\mathrm{MHz}, \mathrm{CDCl}_{3}\right) \delta 211.1,197.8,142.7,134.7,129.4,128.4,127.8,127.1,74.8,51.7,46.1,45.0$, 38.5, 25.1, 23.9; FTIR (neat) 2952, 1684, 1676, 1286; LRMS (CI) calcd for $\left[\mathrm{C}_{15} \mathrm{H}_{16} \mathrm{O}_{3}\right]\left([\mathrm{M}]^{+}\right)$: 244 ; found 244 .

\footnotetext{
${ }^{16}$ Ryu, D. H.; Corey, E. J. J. Am. Chem. Soc. 2003, 125, 6388.
} 


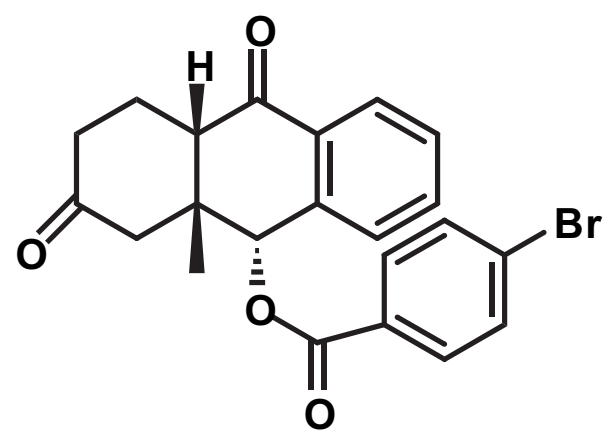

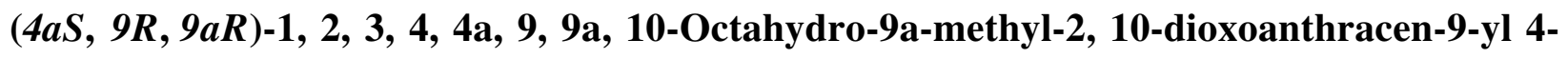
bromobenzoate. To a stirred solution of the alcohol $(30 \mathrm{mg}, 0.12 \mathrm{mmol})$ in pyridine $(0.5 \mathrm{~mL})$ were added $p$-bromobenzoyl chloride $(11.3 \mathrm{mg}, 0.29 \mathrm{mmol})$ and a catalytic amount of 4(dimethylamino) pyridine at $20{ }^{\circ} \mathrm{C}$ and the reaction mixture was stirred for $10 \mathrm{hrs}$ at $65^{\circ} \mathrm{C}$. Pyridine was evaporated in vacuo and water $(4 \mathrm{~mL})$ and dichloromethane $(4 \mathrm{~mL})$ were added. The aqueous layer was extracted with dichloromethane $(4 \mathrm{mLx} 3)$. The combined extract was dried over sodium sulfate and concentrated in vacuo to give pale yellow oily residue. The residue was purified by column chromatography (gradient elution with 10-20\% EtOAc-hexanes) to afford $10 \mathrm{mg}(20 \%)$ of trans product and $31 \mathrm{mg}(59 \%)$ of cis product as a white solid. Recrystallization of cis product from benzene provided colorless crystals suitable for X-ray structure determination: $\mathrm{mp}$ 149.0-150.0 ${ }^{\circ} \mathrm{C}:{ }^{1} \mathrm{H}$ NMR $\left(400 \mathrm{MHz}, \mathrm{CDCl}_{3}\right) \delta 8.16(\mathrm{dd}, 1 \mathrm{H}, J=8.0$, $1.2 \mathrm{~Hz}), 7.91(\mathrm{~d}, 2 \mathrm{H}, J=8.4 \mathrm{~Hz}), 7.58-7.65(\mathrm{~m}, 1 \mathrm{H}), 7.63(\mathrm{~d}, 2 \mathrm{H}, J=8.4 \mathrm{~Hz}), 7.49(\mathrm{dd}, 1 \mathrm{H}, J=$ 8.0, $8.0 \mathrm{~Hz}), 7.29(\mathrm{~d}, 1 \mathrm{H}, J=8.0 \mathrm{~Hz}), 6.49(\mathrm{~s}, 1 \mathrm{H}), 2.83-2.89(\mathrm{~m}, 1 \mathrm{H}), 2.80(\mathrm{t}, 1 \mathrm{H}, J=4.8 \mathrm{~Hz})$, $2.46(\mathrm{~d}, 1 \mathrm{H}, J=14.8 \mathrm{~Hz}), 2.34-2.46(\mathrm{~m}, 2 \mathrm{H}), 2.34(\mathrm{~d}, 1 \mathrm{H}, J=14.8 \mathrm{~Hz}), 2.12-2.20(\mathrm{~m}, 1 \mathrm{H}), 1.24$ $(\mathrm{s}, 3 \mathrm{H}) ;{ }^{13} \mathrm{C} \mathrm{NMR}\left(125 \mathrm{MHz}, \mathrm{CDCl}_{3}\right) \delta 208.8,196.4,165.5,138.7,134.8,132.1$ (x2), 131.4 (x2), 129.9, 129.1, 129.0, 127.8, 127.6, 127.3, 75.5, 51.5, 46.1, 44.4, 38.3, 25.0, 23.2 ; FTIR (neat) 1711, 1721, 1680, 1258, 1097; LRMS (CI) calcd for $\left[\mathrm{C}_{22} \mathrm{H}_{19} \mathrm{BrO}_{4}\right]\left([\mathrm{M}]^{+}\right)$: 426, 428; found 426, 428. 


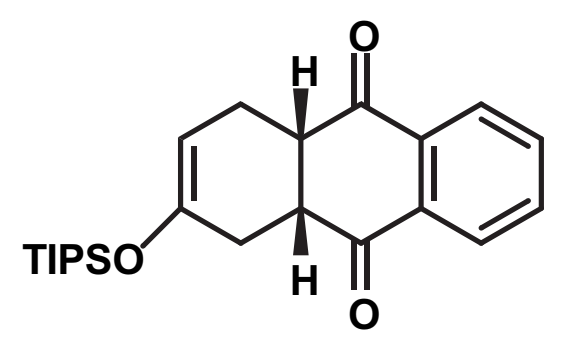

(4aS, 9aR)-1, 4, 4a, 9a-Tetrahydro-2-(triisopropylsilyl)oxy-anthracene-9, 10-dione (Table 2, entry 1). After quenched with $\mathrm{MeOH}-\mathrm{H}_{2} \mathrm{O}$ at $-78^{\circ} \mathrm{C}$ and warmed up to room temperature, the reaction mixture was extracted with hexanes and the combined organic phase was washed with water and brine. After dried over $\mathrm{Na}_{2} \mathrm{SO}_{4}$, the solvent was removed by rotary evaporation and the residue was purified by silica gel chromatography at $-78^{\circ} \mathrm{C}$ to afford $250 \mathrm{mg}(98 \%)$ of the Diels-Alder adduct as colorless oil: ${ }^{1} \mathrm{H}$ NMR $\left(500 \mathrm{MHz}, \mathrm{CDCl}_{3}\right) \delta$ 8.03-8.05 (m, 2H), 7.73-7.76 $(\mathrm{m}, 2 \mathrm{H}), 4.87(\mathrm{~m}, 1 \mathrm{H}), 3.47(\mathrm{dd}, 1 \mathrm{H}, J=11.0,5.5 \mathrm{~Hz}), 3.29-3.31(\mathrm{~m}, 1 \mathrm{H}), 2.63-2.68(\mathrm{~m}, 1 \mathrm{H})$, 2.47-2.53 (m, 1H), 2.25-2.31 (m, 2H), 1.08-1.27(m, 3H), $1.07(\mathrm{~d}, 18 \mathrm{H}, J=7.2 \mathrm{~Hz}) ;{ }^{13} \mathrm{C} \mathrm{NMR}$ $\left(125 \mathrm{MHz}, \mathrm{CDCl}_{3}\right) \delta 198.5,197.6,148.5,134.6,134.5,134.3,134.1,127.12,127.13,100.5$, 47.6, 46.8, 28.7, 24.4, 18.2(x6), 13.0(x3); FTIR (neat) 2941, 2864, 1694, 1187, 881; LRMS (CI) calcd for $\left[\mathrm{C}_{23} \mathrm{H}_{33} \mathrm{O}_{3} \mathrm{Si}\right]\left([\mathrm{MH}]^{+}\right): 385$; found $385 ;[\alpha]_{\mathrm{D}}^{23}+9.2\left(c 1.7, \mathrm{CHCl}_{3}, 97 \%\right.$ ee $)$.

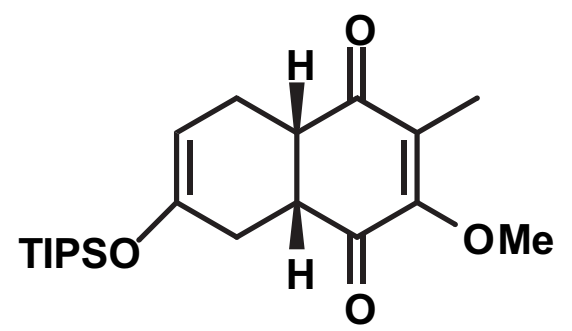

(4aR,8aS)-4a,5,8,8a-Tetrahydro-3-dimethoxy-6-(triisopropylsilyl)oxy-2-

methylnaphthalene-1, 4-dione (Table 2, entry 3). After quenched with $\mathrm{MeOH}-\mathrm{H}_{2} \mathrm{O}$ at $-78^{\circ} \mathrm{C}$ and warmed up to room temperature, the reaction mixture was extracted with hexanes and the combined organic phase was washed with water and brine. After dried over $\mathrm{Na}_{2} \mathrm{SO}_{4}$, the solvent was removed by rotary evaporation and the residue was purified by silica gel chromatography at $-78{ }^{\circ} \mathrm{C}$ to afford $242 \mathrm{mg}(96 \%)$ of an inseparable mixture of Diels-Alder adduct as yellow oil. After reduction with $\mathrm{NaBH}_{4}$ to the corresponding alcohols, the minor diastereomer could be 
separated by silica gel chromatography to afford pure major diastereomer, which was reoxidized by Dess-Martin periodinane to give pure $(4 a R, 8 a S)-4 a, 5,8$, 8a-tetrahydro-3-dimethoxy-6(triisopropylsilyl)oxy-2-methylnaphthalene-1,4-dione as colorless oil: ${ }^{1} \mathrm{H}$ NMR (500 MHz, $\left.\mathrm{CDCl}_{3}\right) \delta 4.79(\mathrm{~m}, 1 \mathrm{H}), 3.94(\mathrm{~s}, 3 \mathrm{H}), 3.25(\mathrm{dd}, 1 \mathrm{H}, J=14.0,7.0 \mathrm{~Hz}), 3.02(\mathrm{~m}, 1 \mathrm{H}), 2.55-2.59$ $(\mathrm{m}, 1 \mathrm{H}), 2.22-2.31(\mathrm{~m}, 1 \mathrm{H}), 2.13-2.20(\mathrm{~m}, 2 \mathrm{H}), 1.86(\mathrm{~s}, 3 \mathrm{H}), 1.02-1.16(\mathrm{~m}, 3 \mathrm{H}), 1.02(\mathrm{~d}, 18 \mathrm{H}, J$ $=7.2 \mathrm{~Hz}) ;{ }^{13} \mathrm{C} \mathrm{NMR}\left(125 \mathrm{MHz}, \mathrm{CDCl}_{3}\right) \delta 200.1,195.2,159.1,148.2,130.8,100.3,60.1,47.1$, 46.6, 27.9, 24.9, 18.2(x6), 12.8(x3), 9.5; FTIR (neat) 2942, 2864, 1673, 1601, 1195, 1126; HRMS (CI) calcd for $\left[\mathrm{C}_{21} \mathrm{H}_{35} \mathrm{O}_{4} \mathrm{Si}\right]\left([\mathrm{MH}]^{+}\right)$: 379.2304; found 379.2299; $[\alpha]_{\mathrm{D}}^{23}+36.8(c$ 2.4, $\mathrm{CHCl}_{3}, 90 \%$ ee).

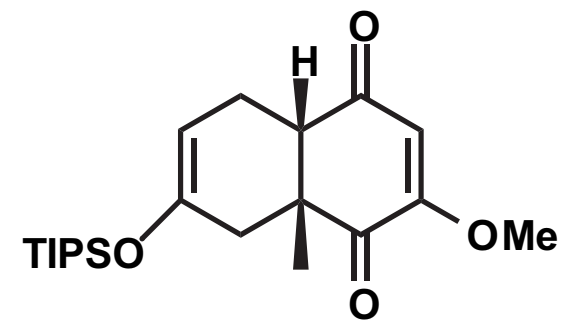

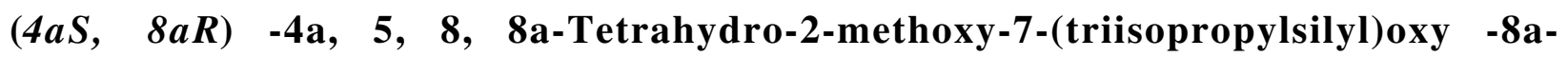
methylnaphthalene-1, 4-dione (Table 2, entry 4). Aqueous work-up and treatment with pentane afforded $247 \mathrm{mg}(98 \%)$ of product as white solid: $\mathrm{mp} 76.0-77.0{ }^{\circ} \mathrm{C}$ (pentane): ${ }^{1} \mathrm{H}$ NMR $\left(400 \mathrm{MHz}, \mathrm{CDCl}_{3}\right) \delta 5.86(\mathrm{~s}, 1 \mathrm{H}), 4.80-4.82(\mathrm{~m}, 1 \mathrm{H}), 3.78(\mathrm{~s}, 3 \mathrm{H}), 2.71(\mathrm{dd}, 1 \mathrm{H}, J=5.6,5.6 \mathrm{~Hz})$, 2.61-2.69 (m, 1H), $2.61(\mathrm{dd}, 1 \mathrm{H}, J=16.8,1.6 \mathrm{~Hz}), 2.18-2.25(\mathrm{~m}, 1 \mathrm{H}), 1.83(\mathrm{~d}, 1 \mathrm{H}, J=16.8 \mathrm{~Hz})$, $1.39(\mathrm{~s}, 3 \mathrm{H}), 1.06-1.16(\mathrm{~m}, 3 \mathrm{H}), 1.04(\mathrm{~d}, 18 \mathrm{H}, J=6.0 \mathrm{~Hz}) ;{ }^{13} \mathrm{C}$ NMR $\left(100 \mathrm{MHz}, \mathrm{CDCl}_{3}\right) \delta 198.3$, 196.3, 160.0, 147.2, 109.8, 99.5, 56.3, 51.3, 48.4, 36.3, 22.8, 22.9, 17.9 (x6), 12.5 (x3); FTIR (neat) 2944, 2862, 1704, 1672, 1604, 1188; LRMS (CI) calcd for $\left[\mathrm{C}_{21} \mathrm{H}_{34} \mathrm{O}_{4} \mathrm{Si}\right]\left([\mathrm{M}]^{+}\right): 378$; found $378 ;[\alpha]_{\mathrm{D}}^{23}-2.4\left(c 1.0, \mathrm{CHCl}_{3}, 92 \%\right.$ ee $)$. 


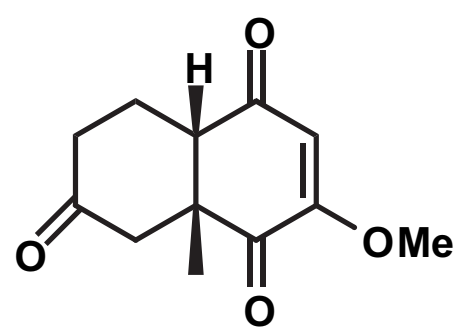

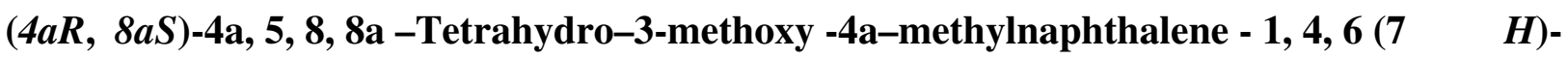
trione. Deprotection of TIPS group afforded $10 \mathrm{mg}(89 \%)$ of ketone product as a white solid: ${ }^{1} \mathrm{H}$ NMR $\left(500 \mathrm{MHz}, \mathrm{CDCl}_{3}\right) \delta 5.99(\mathrm{~s}, 1 \mathrm{H}), 3.82(\mathrm{~s}, 3 \mathrm{H}), 2.91(\mathrm{dd}, 1 \mathrm{H}, J=5.0,5.0 \mathrm{~Hz}), 2.84(\mathrm{~d}, 1 \mathrm{H}$, $J=15.0 \mathrm{~Hz}), 2.32-2.46(\mathrm{~m}, 3 \mathrm{H}), 2.13(\mathrm{~d}, 1 \mathrm{H}, J=15.0 \mathrm{~Hz}), 2.06-2.13(\mathrm{~m}, 1 \mathrm{H}), 1.39(\mathrm{~s}, 3 \mathrm{H}),{ }^{13} \mathrm{C}$ NMR (100 MHz, $\left.\mathrm{CDCl}_{3}\right) \delta 206.1,197.2,194.4,160.1,110.4,56.5,53.2,51.9,47.7,38.3,25.1$, 23.6 ; FTIR (neat) 1709, 1665, 1604, 1228, 1067; LRMS (CI) calcd for $\left[\mathrm{C}_{12} \mathrm{H}_{14} \mathrm{O}_{4}\right]\left([\mathrm{M}]^{+}\right): 222$; found 222 .

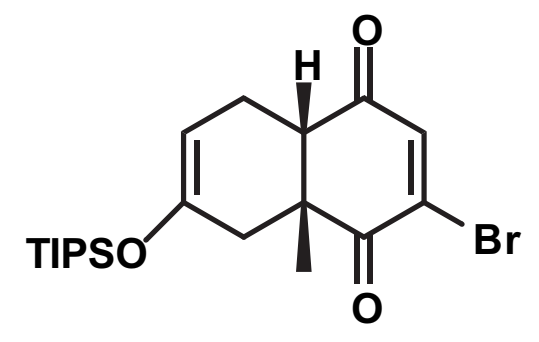

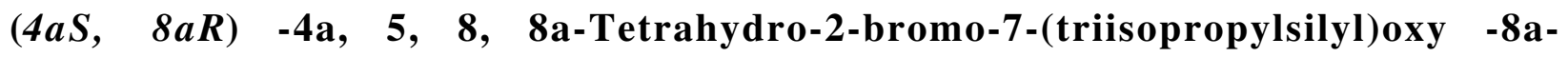
methylnaphthalene-1, 4-dione (Table 2, entry 5). Aqueous work-up afforded $270 \mathrm{mg}(95 \%)$ of product as a pale yellow oil: ${ }^{1} \mathrm{H}$ NMR $\left(400 \mathrm{MHz}, \mathrm{CDCl}_{3}\right) \delta 7.18(\mathrm{~s}, 1 \mathrm{H}), 4.77-4.79(\mathrm{~m}, 1 \mathrm{H}), 2.78$ $(\mathrm{dd}, 1 \mathrm{H}, J=5.4,5.4 \mathrm{~Hz}), 2.59-2.67(\mathrm{~m}, 1 \mathrm{H}), 2.52(\mathrm{dd}, 1 \mathrm{H}, J=17.2,2.0 \mathrm{~Hz}), 2.16-2.23(\mathrm{~m}, 1 \mathrm{H})$, $1.84(\mathrm{~d}, 1 \mathrm{H}, J=17.6 \mathrm{~Hz}), 1.40(\mathrm{~s}, 3 \mathrm{H}), 1.04-1.13(\mathrm{~m}, 3 \mathrm{H}), 1.04(\mathrm{~d}, 18 \mathrm{H}, J=6.8 \mathrm{~Hz}) ;{ }^{13} \mathrm{C} \mathrm{NMR}$ $\left(100 \mathrm{MHz} \mathrm{CDCl}_{3}\right) \delta 195.8,193.7,147.1,140.5,139.8,99.0,51.7,48.4,36.7,22.9,22.1,17.8$ (x6), 12.4 (x3); FTIR (neat) 2942, 2865, 1690, 1208; LRMS (CI) calcd for $\left[\mathrm{C}_{20} \mathrm{H}_{32} \mathrm{BrO}_{3} \mathrm{Si}\right]$ $\left([\mathrm{MH}]^{+}\right): 427,429$; found 427,429; $[\alpha]_{\mathrm{D}}^{23}-14.3\left(c 1.0, \mathrm{CHCl}_{3}, 91 \%\right.$ ee $)$. 


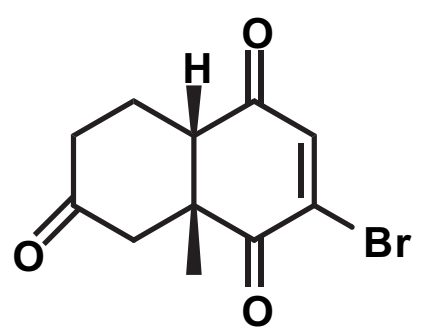

(4aR, $8 a S)$-3-Bromo-4a, 5, 8, 8a-tetrahydro-4a-methylnaphthalene-1, 4, 6(7H)-trione.

Deprotection of TIPS group and recrystallization from a mixture solvent (EtOAc: Hexanes=1:7) afforded $56 \mathrm{mg}(65 \%)$ of ketone product as golden crystals suitable for X-ray structure determination: mp $113-114{ }^{\circ} \mathrm{C} ;{ }^{1} \mathrm{H}$ NMR $\left(400 \mathrm{MHz}, \mathrm{CDCl}_{3}\right) \delta 7.32(\mathrm{~s}, 1 \mathrm{H}), 3.00(\mathrm{dd}, 1 \mathrm{H}, J=$ 5.6, 5.6 Hz), $2.80(\mathrm{~d}, 1 \mathrm{H}, J=14.8 \mathrm{~Hz}), 2.29-2.48(\mathrm{~m}, 3 \mathrm{H}), 2.16(\mathrm{~d}, 1 \mathrm{H}, J=14.8 \mathrm{~Hz}), 2.04-2.14$ (m, 1H), $1.41(\mathrm{~s}, 3 \mathrm{H}) ;{ }^{13} \mathrm{C} \mathrm{NMR}\left(100 \mathrm{MHz}, \mathrm{CDCl}_{3}\right) \delta 205.2,195.3,192.1,140.8,140.1,53.7$, 51.9, 48.0, 38.0, 24.6, 24.0; FTIR (neat) 2932, 1683, 1588, 1181; LRMS (CI) calcd for $\left[\mathrm{C}_{11} \mathrm{H}_{11} \mathrm{BrO}_{3}\right]\left([\mathrm{M}]^{-}\right): 270,272$; found 270, 272; $[\alpha]_{\mathrm{D}}^{23}-12.8\left(c 1.0, \mathrm{CHCl}_{3},>91 \%\right.$ ee $)$.

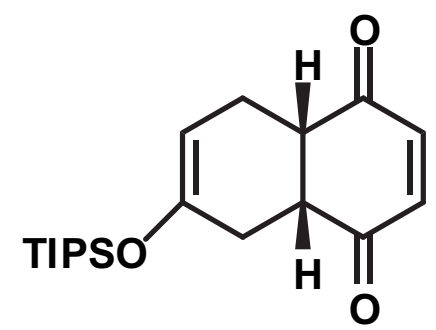

(4aR, 8aS)-4a, 5, 8, 8a-Tetrahydro-6-(triisopropylsilyl)oxy-naphthalene-1, 4-dione (Table 3, entry 1). After quenched with $\mathrm{MeOH}-\mathrm{H}_{2} \mathrm{O}$ at $-78^{\circ} \mathrm{C}$ and warmed up to room temperature, the reaction mixture was extracted with hexanes and the combined organic phase was washed with water and brine. After dried over $\mathrm{Na}_{2} \mathrm{SO}_{4}$, the solvent was removed by rotary evaporation and the residue was purified by silica gel chromatography at $-78^{\circ} \mathrm{C}$ to afford $190 \mathrm{mg}(85 \%)$ of the DielsAlder adduct as a yellow oil: ${ }^{1} \mathrm{H}$ NMR $\left(400 \mathrm{MHz} \mathrm{CDCl}_{3}\right) \delta 6.69(\mathrm{~d}, 1 \mathrm{H}, J=10.4 \mathrm{~Hz}), 6.64(\mathrm{~d}$, $1 \mathrm{H}, J=10.4 \mathrm{~Hz}), 4.83(\mathrm{~m}, 1 \mathrm{H}), 3.30(\mathrm{dd}, 1 \mathrm{H}, J=12.0,6.0 \mathrm{~Hz}), 3.12(\mathrm{dd}, 1 \mathrm{H}, J=12.0,6.0 \mathrm{~Hz})$, 2.53-2.60 (m, 1H), 2.43-2.49 (m, 1H), 2.18-2.24 (m, 2H), 0.88-1.25 (m, 3H), $1.05(\mathrm{~d}, 18 \mathrm{H}, J=$ $6.6 \mathrm{~Hz}) ;{ }^{13} \mathrm{C}$ NMR $\left(100 \mathrm{MHz}, \mathrm{CDCl}_{3}\right) \delta 200.3,199.2,148.3,139.6,139.4,100.3,47.4,46.5,28.4$, 
24.2, 18.1(x6), 12.8 (x3); FTIR (neat) 2864, 1684, 1653, 1194, 881; $[\alpha]_{\mathrm{D}}^{23}+18.0$ (c 1.8, $\mathrm{CHCl}_{3}$, $88 \%$ ee).

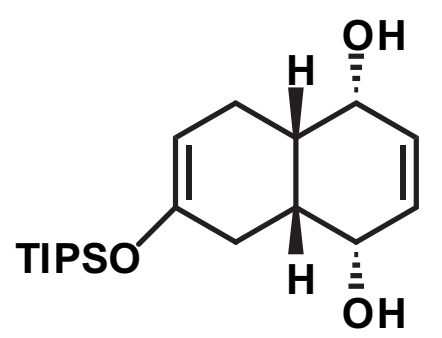

$(1 R, 4 S, 4 a R, 8 a S)$-1, 4, 4a, 5, 8, 8a-hexahydro-6-(triisopropylsilyl)oxy-naphthalene-1, 4diol. To a stirred solution of the (triisopropylsilyl)oxy quinone adduct $(66.8 \mathrm{mg}, 0.20 \mathrm{mmol}$ ) in $\mathrm{MeOH}(5 \mathrm{~mL})$ was added a $\mathrm{CeCl}_{3} .7 \mathrm{H}_{2} \mathrm{O}(59.2 \mathrm{mg}, 0.24 \mathrm{mmol})$ and sodium borohydride $(9.1 \mathrm{mg}$, $0.24 \mathrm{mmol})$ at $0{ }^{\circ} \mathrm{C}$ and the reaction mixture was stirred for 10 min at $0{ }^{\circ} \mathrm{C}$. Water $(4 \mathrm{~mL})$ and hexanes $(4 \mathrm{~mL})$ were added and the organic layers were separated. The aqueous layer was extracted with hexanes and ether $(10 \mathrm{ml}$ x 3). The combined extract was dried over sodium sulfate and concentrated in vacuo to give pale yellow oil, which was purified by silica gel chromatography to afford diol as a colorless oil (45.4 mg, 68\%): ${ }^{1} \mathrm{H}$ NMR (400 MHz, $\left.\mathrm{CDCl}_{3}\right) \delta$ $5.74(\mathrm{~s}, 2 \mathrm{H}), 4.94(\mathrm{~m}, 1 \mathrm{H}), 4.24(\mathrm{~m}, 2 \mathrm{H}), 2.09-2.31(\mathrm{~m}, 6 \mathrm{H}), 1.01-1.24(\mathrm{~m}, 3 \mathrm{H}), 1.04(\mathrm{~d}, 18 \mathrm{H}, J=$ $6.6 \mathrm{~Hz}) ;{ }^{13} \mathrm{C} \mathrm{NMR}\left(100 \mathrm{MHz}, \mathrm{CDCl}_{3}\right) \delta 152.0,129.9,129.8,101.9,68.7,68.4,35.4,34.2,29.9$, 23.6, 18.2 (x6), 12.9 (x3); FTIR (neat) 2863, 1662, 1193, 677; LRMS (CI) calcd for $\left[\mathrm{C}_{19} \mathrm{H}_{34} \mathrm{O}_{3} \mathrm{Si}\right]$ ([M-H] $\left.]^{-}\right): 337$; found 337; $[\alpha]_{\mathrm{D}}^{23}-15.0\left(c 0.8, \mathrm{CHCl}_{3}\right)$.

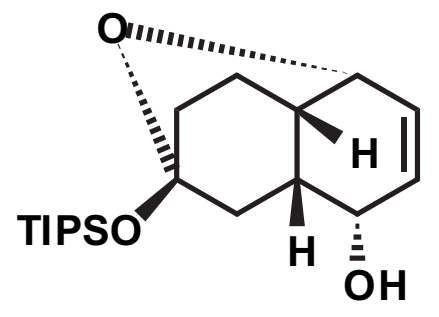

\section{TIPS cyclic ketal.}

To a stirred solution of diol obtained previously $(33.8 \mathrm{mg}, 0.10 \mathrm{mmol})$ in $5 \mathrm{~mL}$ of THF, $\mathrm{BF}_{3} \bullet \mathrm{OEt}_{2}(2.8 \mathrm{mg}, 0.02 \mathrm{mmol})$ was added by syringe at $-78^{\circ} \mathrm{C}$. The mixture was stirred at $-78{ }^{\circ} \mathrm{C}$ 
for $2 \mathrm{~h}$ and then diluted with $10 \mathrm{~mL}$ of diethyl ether and $5 \mathrm{~mL}$ of saturated aqueous $\mathrm{NaHCO}_{3}$. The mixture was warmed up to room temperature and the two phases were separated. The aqueous phase was extracted with diethyl ether $(2 \times 10 \mathrm{~mL})$ and the combined organic phase was washed with saturated aqueous $\mathrm{NaCl}$, dried over $\mathrm{Na}_{2} \mathrm{CO}_{3}$. The solvent was removed by rotary evaporation and the residue was purified by silica gel chromatography to afford $28.7 \mathrm{mg}(85 \%)$ of ketal as a colorless oil: ${ }^{1} \mathrm{H}$ NMR $\left(400 \mathrm{MHz}, \mathrm{CDCl}_{3}\right) \delta 6.00$ (ddd, $\left.1 \mathrm{H}, J=9.6,5.2,2.0 \mathrm{~Hz}\right), 5.65$ $(\mathrm{dt}, 1 \mathrm{H}, J=9.6,1.6 \mathrm{~Hz}), 4.34(\mathrm{~m}, 1 \mathrm{H}), 4.10(\mathrm{~m}, 1 \mathrm{H}), 2.40(\mathrm{~m}, 1 \mathrm{H}), 1.52-1.96(\mathrm{~m}, 7 \mathrm{H}), 1.01-1.08$ (m, 3H), $1.03(\mathrm{~d}, 18 \mathrm{H}, J=6.6 \mathrm{~Hz}) ;{ }^{13} \mathrm{C} \mathrm{NMR}\left(100 \mathrm{MHz}, \mathrm{CDCl}_{3}\right) \delta 131.5,131.2,96.6,70.9,70.3$, 37.1, 35.1, 33.2, 29.9, 25.4, 18.4 (x6), 13.3 (x3); FTIR (neat) 2940, 2863, 1339, 1180, 994; LRMS (CI) calcd for $\left[\mathrm{C}_{19} \mathrm{H}_{35} \mathrm{O}_{3} \mathrm{Si}\right]\left([\mathrm{MH}]^{+}\right)$: 339; found 339; $[\alpha]_{\mathrm{D}}^{23}-108\left(c 1.9, \mathrm{CHCl}_{3}\right)$.

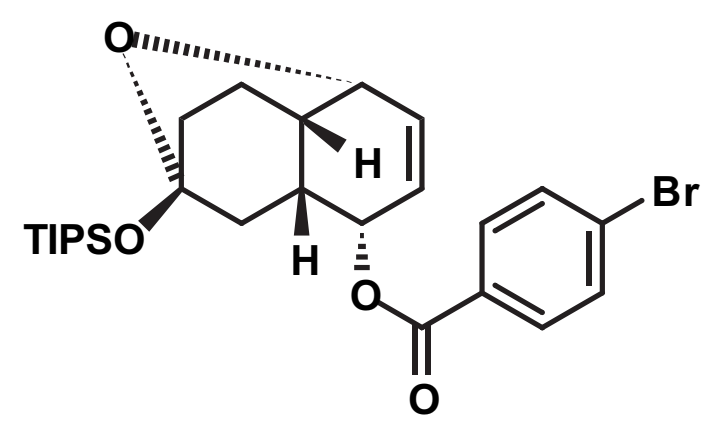

\section{p-Bromobenzoate.}

Protection of monoalcohol obtained previously with $p$-bromobenzoyl chloride afforded bromobenzoate $(87 \%)$ as a colorless oil: ${ }^{1} \mathrm{H}$ NMR $\left(400 \mathrm{MHz}, \mathrm{CDCl}_{3}\right) \delta 7.87(\mathrm{~d}, 2 \mathrm{H}, J=6.4 \mathrm{~Hz})$, $7.58(\mathrm{~d}, 2 \mathrm{H}, J=6.4 \mathrm{~Hz}), 6.13(\mathrm{ddd}, 1 \mathrm{H}, J=9.6,4.8,2.4 \mathrm{~Hz}), 5.73(\mathrm{dt}, 1 \mathrm{H}, J=9.6,1.2 \mathrm{~Hz}), 5.63$ (m, 1H), 4.19 (m, 1H), 2.65 (m, 1H), $2.15(\mathrm{~m}, 1 \mathrm{H}), 1.59-1.99$ (m, 6H), 1.01-1.10 (m, 3H), 1.04 $(\mathrm{d}, 18 \mathrm{H}, J=6.6 \mathrm{~Hz}) ;{ }^{13} \mathrm{C}$ NMR $\left(100 \mathrm{MHz}, \mathrm{CDCl}_{3}\right) \delta 165.6,133.2,132.0(\mathrm{x} 2), 131.4(\mathrm{x} 2), 129.4$, 128.4, 127.3, 96.3, 74.1, 70.1, 35.6, 34.3, 33.0, 29.7, 25.3, 18.5 (x6), 13.3 (x3); FTIR (neat) 2922, 2862, 1719, 1265, 995; LRMS (CI) calcd for $\left[\mathrm{C}_{26} \mathrm{H}_{37} \mathrm{BrO}_{4} \mathrm{Si}\right]\left([\mathrm{M}]^{+}\right)$: 520, 522; found 520, $522 ;[\alpha]_{\mathrm{D}}^{23}-43.8\left(c 2.6, \mathrm{CHCl}_{3}\right)$. 


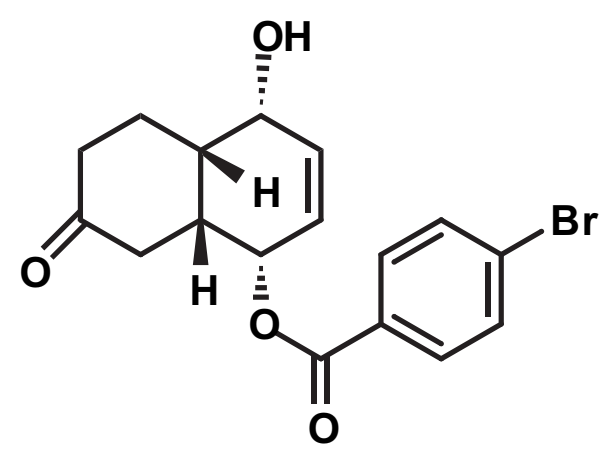

$(1 R, 4 S, 4 a R, 8 a S)-1,4,4 a, 5,6,7,8,8 \mathrm{a}-$ Octahydro- 1-hydroxy-6-oxonaphthalen-4-yl-4bromobenzoate. After desilylation with $10 \% \mathrm{HF}\left(\mathrm{CH}_{3} \mathrm{CN}\right)$, residue was purified by silica gel chromatography to afford the hydroxyketone as a white solid. Recrystallization from a mixture solvent (diethyl ether-hexanes $2: 1$ ) at $0{ }^{\circ} \mathrm{C}$ for 2 days provided the colorless crystal suitable for the X-ray structure determination: $\mathrm{mp} 138-139{ }^{\circ} \mathrm{C}:{ }^{1} \mathrm{H} \mathrm{NMR}\left(400 \mathrm{MHz}, \mathrm{CDCl}_{3}\right) \delta 7.69(\mathrm{~d}, 2 \mathrm{H}$, $J=6.8 \mathrm{~Hz}), 7.54(\mathrm{~d}, 2 \mathrm{H}, J=6.8 \mathrm{~Hz}), 5.82-5.86(\mathrm{~m}, 2 \mathrm{H}), 5.62(\mathrm{~m}, 1 \mathrm{H}), 4.56(\mathrm{dd}, 1 \mathrm{H}, J=6.4,1.2$ $\mathrm{Hz}), 2.07-2.65(\mathrm{~m}, 8 \mathrm{H}) ;{ }^{13} \mathrm{C}$ NMR $\left(100 \mathrm{MHz}, \mathrm{CDCl}_{3}\right) \delta 209.8,171.4,165.5,134.4,131.9,131.4$, 128.7, 128.6, 124.5, 69.1, 68.9, 42.9, 40.3, 37.7, 37.2, 21.0; FTIR (neat) 1722, 1182, 1025, 909 , 730; LRMS (CI) calcd for $\left[\mathrm{C}_{17} \mathrm{H}_{17} \mathrm{BrO}_{4}\right]\left([\mathrm{M}]^{+}\right)$: 364, 366; found 364, 366.

(4aS, 8aR)-4a, 5, 8, 8a-Tetrahydro-7-(triisopropylsilyl)oxy-2-phenylnaphthalene-1, 4-dione

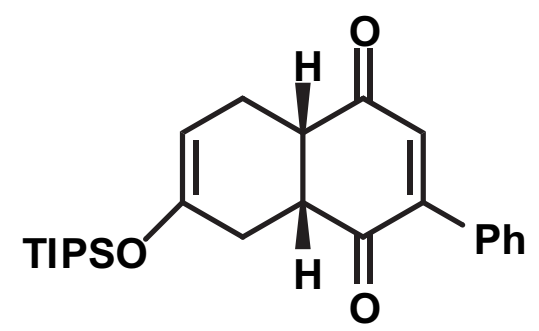

(Table 3, entry 2). After quenched with $\mathrm{MeOH}-\mathrm{H}_{2} \mathrm{O}$ at $-78{ }^{\circ} \mathrm{C}$ and warmed up to room temperature, the reaction mixture was extracted with hexanes and the combined organic phase was washed with water and brine. After dried over $\mathrm{Na}_{2} \mathrm{SO}_{4}$, the solvent was removed by rotary evaporation and the residue was purified by silica gel chromatography at $-78{ }^{\circ} \mathrm{C}$ to afford $246 \mathrm{mg}$ 
(90\%) of the Diels-Alder adduct as a colorless oil: ${ }^{1} \mathrm{H}$ NMR (400 MHz, $\left.\mathrm{CDCl}_{3}\right) \delta$ 7.43-7.48 (m, 5H), $6.77(\mathrm{~s}, 1 \mathrm{H}), 4.86(\mathrm{~m}, 1 \mathrm{H}), 3.52(\mathrm{dd}, 1 \mathrm{H}, J=9.6,4.4 \mathrm{~Hz}), 3.18(\mathrm{dd}, 1 \mathrm{H}, J=10.0,4.4 \mathrm{~Hz})$, 2.66-2.71 (m, 1H), 2.47-2.53 (m, 1H), 2.52-2.30 (m, 2H), 1.12-1.31 (m, 3H), $1.08(\mathrm{~d}, 18 \mathrm{H}, \mathrm{J}=$ $6.5 \mathrm{~Hz}) ;{ }^{13} \mathrm{C}$ NMR $\left(100 \mathrm{MHz}, \mathrm{CDCl}_{3}\right) \delta 200.2,199.0,149.53,148.4,134.8,133.3,130.6,129.5$, 129.3, 128.8(x2), 100.3, 48.8, 46.7, 28.4, 24.5, 18.2(x6), 12.8(x3); FTIR (neat) 2941, 2863, 1673, 1189, 881; HRMS (CI) calcd for $\left[\mathrm{C}_{25} \mathrm{H}_{35} \mathrm{O}_{3} \mathrm{Si}\right]\left([\mathrm{MH}]^{+}\right): 411.2355$; found 411.2352; $[\alpha]_{\mathrm{D}}^{23}$ $+47.8\left(\right.$ ( $2.9, \mathrm{CHCl}_{3}, 94 \%$ ee $)$.

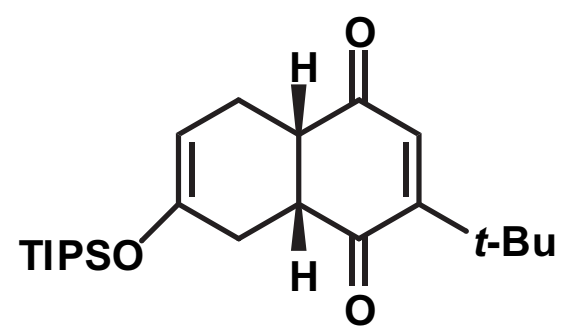

(4aS, 8aR)-2-tert-Butyl-4a, 5, 8, 8a-tetrahydro-7-(triisopropylsilyl)oxy-naphthalene-1, 4dione (Table 3, entry 3). After quenched with $\mathrm{MeOH}-\mathrm{H}_{2} \mathrm{O}$ at $-78^{\circ} \mathrm{C}$ and warmed up to room temperature, the reaction mixture was extracted with hexanes and the combined organic phase was washed with water and brine. After dried over $\mathrm{Na}_{2} \mathrm{SO}_{4}$, the solvent was removed by rotary evaporation and the residue was purified by silica gel chromatography at $-78^{\circ} \mathrm{C}$ to afford 238 mg $(92 \%)$ of the Diels-Alder adduct as a colorless oil: ${ }^{1} \mathrm{H}$ NMR $\left(400 \mathrm{MHz}, \mathrm{CDCl}_{3}\right) \delta 6.46(\mathrm{~s}$, $1 \mathrm{H}), 4.82(\mathrm{~m}, 1 \mathrm{H}), 3.25(\mathrm{dd}, 1 \mathrm{H}, J=11.2,5.6 \mathrm{~Hz}), 3.03(\mathrm{dd}, 1 \mathrm{H}, J=12.4,6.0 \mathrm{~Hz}), 2.47-2.53(\mathrm{~m}$, 1H), 2.36-2.42 (m, 1H), 2.12-2.21 (m, 2H), $1.24(\mathrm{~s}, 9 \mathrm{H}), 1.12-1.32(\mathrm{~m}, 3 \mathrm{H}), 1.09$ (d, 18H, J = 6.6 Hz); 13C NMR (100 MHz, CDCl3) $\delta 201.0,199.9,160.6,148.4$, 133.6, 100.4, 49.3, 46.4, 30.1, 29.0(x3), 28.4, 24.2, 18.2(x6), 12.8(x3); FTIR (neat) 2942, 2865, 1675, 1212, 753; HRMS (CI) calcd for $\left[\mathrm{C}_{23} \mathrm{H}_{39} \mathrm{O}_{3} \mathrm{Si}\right]\left([\mathrm{MH}]^{+}\right)$: 391.2668; found 391.2670; $[\alpha]_{\mathrm{D}}^{23}+23.9\left(\right.$ c 2.2, $\mathrm{CHCl}_{3}, 91 \%$ ee). 


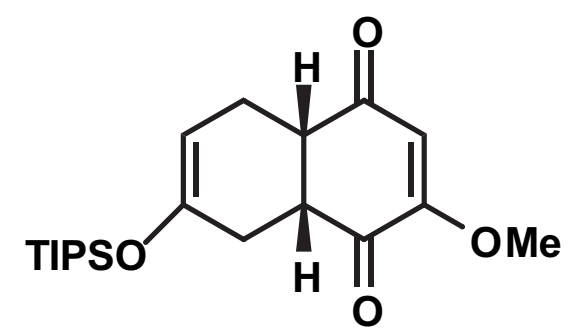

$(4 a S, 8 a R)$-4a, 5, 8, 8a-Tetrahydro-2-methoxy-7-(triisopropylsilyloxy)-naphthalene-1, 4dione (Table 3, entry 4). Aqueous work-up and treatment with pentane afforded $230 \mathrm{mg}(95 \%)$ of product as a white solid: mp 79.0-80.0 ${ }^{\circ} \mathrm{C}$ (pentane): ${ }^{1} \mathrm{H}$ NMR $\left(400 \mathrm{MHz}, \mathrm{CDCl}_{3}\right) \delta 5.87(\mathrm{~s}$, $1 \mathrm{H}), 4.81-4.83(\mathrm{~m}, 1 \mathrm{H}), 3.78(\mathrm{~s}, 3 \mathrm{H}), 3.32(\mathrm{dd}, 1 \mathrm{H}, J=12.0,5.2 \mathrm{~Hz}), 3.04(\mathrm{dd}, 1 \mathrm{H}, J=13.2,6.0$ Hz), 2.60-2.66 (m, 1H), 2.38-2.46 (m, 1H), 2.18-2.29 (m, 2H), 1.10-1.18 (m, 3H), 1.05 (d, 18H, $J$ $=6.4 \mathrm{~Hz}) ;{ }^{13} \mathrm{C} \mathrm{NMR}\left(100 \mathrm{MHz}, \mathrm{CDCl}_{3}\right) \delta 199.3,193.7,161.3,147.9,110.2,100.1,56.3,46.6$, 45.8, 27.9, 24.6, 17.9 (x6), 12.5 (x3); FTIR (neat) 2941, 2865, 1701, 1672, 1598, 1184; LRMS (CI) calcd for $\left[\mathrm{C}_{20} \mathrm{H}_{32} \mathrm{O}_{4} \mathrm{Si}\right]\left([\mathrm{M}]^{+}\right)$: 364; found $364 ;[\alpha]_{\mathrm{D}}^{23}+83.6\left(c 1.0, \mathrm{CHCl}_{3}, 91 \%\right.$ ee $)$.

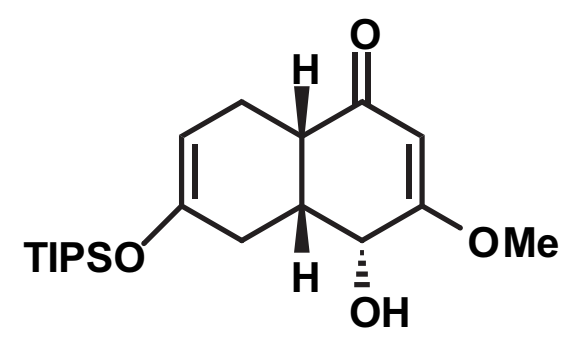

(4R,4aR,8aS)-4a,5,8,8a-Tetrahydro-4-hydroxy-3-methoxynaphthalene-1,6(4H,7H)-dione.

Selective reduction of quinone with sodium borohydride afforded crude mono alcohol product as a pale yellow solid; ${ }^{1} \mathrm{H}$ NMR $\left(500 \mathrm{MHz}, \mathrm{CDCl}_{3}\right) \delta 5.36(\mathrm{~s}, 1 \mathrm{H}), 4.84-4.85(\mathrm{~m}, 1 \mathrm{H}), 4.81(\mathrm{~d}, 1 \mathrm{H}, J$ $=5.0 \mathrm{~Hz}), 3.77(\mathrm{~s}, 3 \mathrm{H}), 2.81-2.88(\mathrm{~m}, 2 \mathrm{H}), 2.51-2.53(\mathrm{~m}, 1 \mathrm{H}), 2.19(\mathrm{dd}, 1 \mathrm{H}, J=18.0,5.0 \mathrm{~Hz})$, 2.03-2.14 (m, 1H), 1.06-1.15 (m, 3H), $1.04(\mathrm{~d}, 9 \mathrm{H}, J=7.0 \mathrm{~Hz}), 1.03(\mathrm{~d}, 9 \mathrm{H}, J=7.0 \mathrm{~Hz})$. 


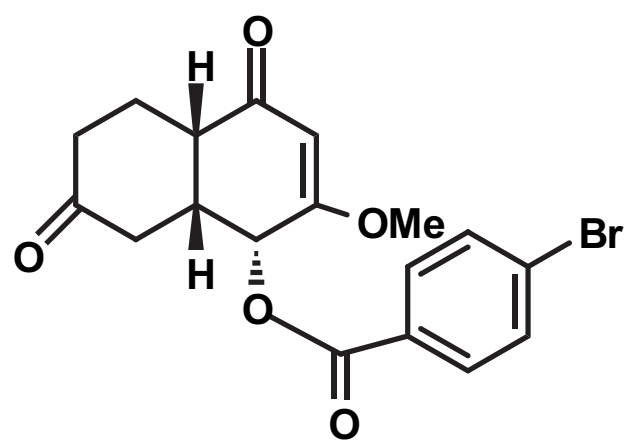

$(4 R, 4 a R, 8 a S)-1,4,4 a, 5,6,7,8,8 a-O c t a h y d r o-3-m e t h o x y-1$, 6-dioxonaphthalen-4-yl 4bromobenzoate. $p$-Bromobenzoylation of the alcohol at $20^{\circ} \mathrm{C}$ and deprotection of TIPS group provided crude ketone product. The crude product was purified by column chromatography (gradient elution with 30-60\% EtOAc-hexanes) to afford $30 \mathrm{mg}$ (48\% for two steps) of product as a white solid. Recrystallization from a mixture solvent (dichloromethane: hexanes=1:5) provided colorless crystals suitable for X-ray structure determination: mp 191-192 ${ }^{\circ} \mathrm{C}:{ }^{1} \mathrm{H}$ NMR $\left(400 \mathrm{MHz}, \mathrm{CDCl}_{3}\right) \delta 7.86(\mathrm{~d}, 2 \mathrm{H}, J=8.4 \mathrm{~Hz}), 7.61(\mathrm{~d}, 2 \mathrm{H}, J=8.4 \mathrm{~Hz}), 6.26(\mathrm{~d}, 1 \mathrm{H}, J=4.8 \mathrm{~Hz})$, $5.55(\mathrm{~s}, 1 \mathrm{H}), 3.73(\mathrm{~s}, 3 \mathrm{H}), 3.03(\mathrm{dt}, 1 \mathrm{H}, J=15.2,5.2 \mathrm{~Hz}), 2.85(\mathrm{dd}, 1 \mathrm{H}, J=9.6,4.8 \mathrm{~Hz}), 2.74$ (ddd, 1H, $J=13.6,10.8,5.2 \mathrm{~Hz}), 2.57(\mathrm{dd}, 1 \mathrm{H}, J=14.8,5.2 \mathrm{~Hz}), 2.39-2.52(\mathrm{~m}, 2 \mathrm{H}), 2.34(\mathrm{dt}$, $1 \mathrm{H}, J=14.8,5.2 \mathrm{~Hz}), 1.80-1.89(\mathrm{~m}, 1 \mathrm{H}) ;{ }^{13} \mathrm{C} \mathrm{NMR}\left(100 \mathrm{MHz}, \mathrm{CDCl}_{3}\right) \delta 208.7,196.7,170.6$, 164.8, 131.9 (x2), 131.3 (x2), 128.9, 127.8, 102.6, 69.3, 56.6, 44.1, 40.3, 39.2, 38.4, 25.4 ; FTIR (neat) 2944, 1719, 1654, 1607, 1584, 1199, 1007; LRMS (CI) calcd for $\left[\mathrm{C}_{18} \mathrm{H}_{17} \mathrm{BrO}_{5}\right]\left([\mathrm{M}]^{+}\right)$: 392, 394; found 392, 394. 

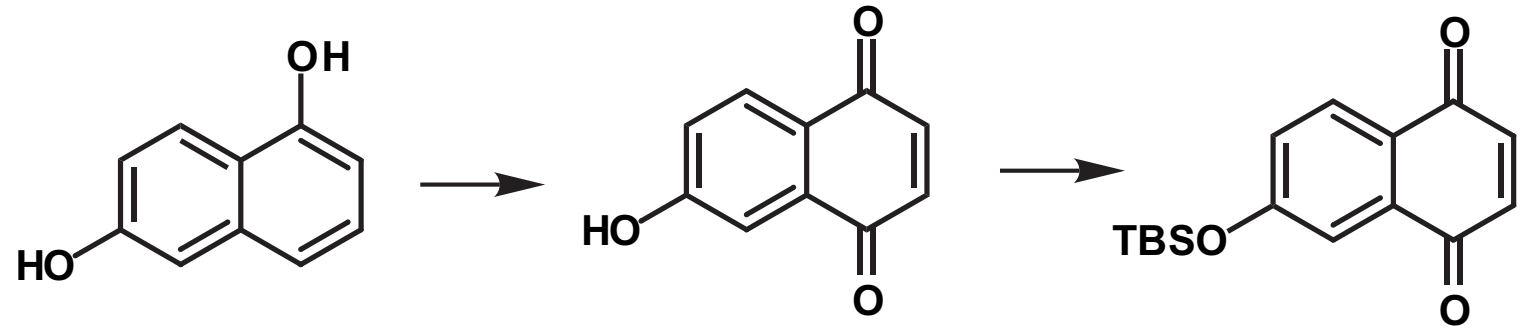

6-Hydroxynaphthalene-1, 4-dione : Oxygen was bubbled rapidly into a stirring solution of naphthalene-1,6-diol (3 g, $18.7 \mathrm{~mol}$ ) and bis(salicylidene)ethylenediaminocobalt (II) hydrate (salcomine, $0.3 \mathrm{~g}$ ) in anhydrous DMF (20ml) for $10 \mathrm{~h}$. The reaction mixture was passed through a Celite pad and the filtrate was concentrated in vacuo to give gummy residue. Water (100mL) and EtOAc (20mL) were added and the aqueous layer was extracted with EtOAc (4x 20mL). The combined extract was dried over anhydrous $\mathrm{Na}_{2} \mathrm{SO}_{4}$ and concentrated to afford black red solid residue. The crude product was purified by column chromatography (gradient elution with $30-60 \%$ EtOAc-hexanes) to afford $2.3 \mathrm{~g}$ (67\% yield) of product as orange solid: ${ }^{1} \mathrm{H}$ NMR (500 $\left.\mathrm{MHz}, \mathrm{CDCl}_{3}\right) \delta 8.04(\mathrm{~d}, 1 \mathrm{H}, J=8.5 \mathrm{~Hz}), 7.47(\mathrm{~d}, 1 \mathrm{H}, J=2.5 \mathrm{~Hz}), 7.18(\mathrm{dd}, 1 \mathrm{H}, J=8.5,2.5 \mathrm{~Hz})$, $6.94(\mathrm{~s}, 2 \mathrm{H}), 5.51(\mathrm{~s}, 1 \mathrm{H})$; LRMS (CI) calcd for $\left[\mathrm{C}_{10} \mathrm{H}_{6} \mathrm{O}_{3}\right]\left([\mathrm{M}-\mathrm{H}]^{-}\right)$: 173; found173.

6- $t$-Butyldimethyl silyloxy naphthalene-1, 4-dione : To a stirred solution of the phenol (530 $\mathrm{mg}, 3.0 \mathrm{mmol})$ in anhydrous DMF $(6 \mathrm{~mL})$ were added imidazole $(576 \mathrm{mg}, 9.13 \mathrm{mmol})$ and $t$ butyldimethylsilyl chloride $(688 \mathrm{mg}, 4.56 \mathrm{mmol})$ at $20{ }^{\circ} \mathrm{C}$ and the reaction mixture was stirred for $20 \mathrm{~min}$ at $20^{\circ} \mathrm{C}$. Water $(60 \mathrm{~mL})$ and diethyl ether $(10 \mathrm{~mL})$ were added. The aqueous layer was extracted with diethyl ether $(10 \mathrm{mLx} 3)$. The combined extract was dried over sodium sulfate and concentrated in vacuo to give brown oily residue. The residue was purified by column chromatography (gradient elution with 1-3\% EtOAc-hexanes) to afford $319 \mathrm{mg}$ (36\%) of TBS ether product as green oil: ${ }^{1} \mathrm{H}$ NMR $\left(400 \mathrm{MHz}, \mathrm{CDCl}_{3}\right) \delta 8.00(\mathrm{~d}, 1 \mathrm{H}, J=8.4 \mathrm{~Hz}), 7.45(\mathrm{~d}, 1 \mathrm{H}, J$ $=2.4 \mathrm{~Hz}), 7.15(\mathrm{dd}, 1 \mathrm{H}, J=8.4,2.4 \mathrm{~Hz}), 6.92(\mathrm{~d}, 2 \mathrm{H}, J=0.8 \mathrm{~Hz}), 1.00(\mathrm{~s}, 9 \mathrm{H}), 0.27(\mathrm{~s}, 6 \mathrm{H}) ;{ }^{13} \mathrm{C}$ 
NMR $\left(125 \mathrm{MHz}, \mathrm{CDCl}_{3}\right) \delta 185.1,184.1,161.1,138.9,138.3,133.9,129.0,125.9,125.4,116.9$, 25.5 (x3), 18.2, -4.4 (x2) ; FTIR (neat) 2857, 1664, 1589, 1305, 1239; LRMS (CI) calcd for $\left[\mathrm{C}_{16} \mathrm{H}_{21} \mathrm{O}_{3} \mathrm{Si}\right]\left([\mathrm{MH}]^{+}\right)$: 289; found 289.

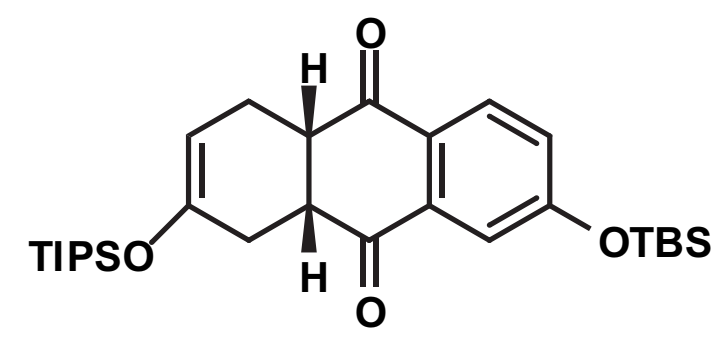

(4 a R, 9aS)-2-(Triisopropylsilyl)oxy-1，4，4a，9a-tetrahydro-7-t-butyldimethylsilyloxy anthracene-9, 10-dione. Aqueous work-up and silica gel filtration afforded $342 \mathrm{mg}$ (95\%) of product as pale orange oil: ${ }^{1} \mathrm{H} \mathrm{NMR}\left(500 \mathrm{MHz}, \mathrm{CDCl}_{3}\right) \delta 7.96(\mathrm{~d}, 1 \mathrm{H}, J=8.0 \mathrm{~Hz}), 7.38(\mathrm{~d}, 1 \mathrm{H}$, $J=2.5 \mathrm{~Hz}), 7.12(\mathrm{dd}, 1 \mathrm{H}, J=8.0,2.5 \mathrm{~Hz}), 4.84-4.85(\mathrm{~m}, 1 \mathrm{H}), 3.43(\mathrm{dd}, 1 \mathrm{H}, J=12.0,5.5 \mathrm{~Hz})$, $3.04(\mathrm{dt}, 1 \mathrm{H}, J=7.5,5.5 \mathrm{~Hz}), 2.64-2.70(\mathrm{~m}, 1 \mathrm{H}), 2.42-2.47(\mathrm{~m}, 1 \mathrm{H}), 2.23-2.27(\mathrm{~m}, 2 \mathrm{H}), 1.08-$ $1.17(\mathrm{~m}, 3 \mathrm{H}), 1.05(\mathrm{~d}, 18 \mathrm{H}, J=7.0 \mathrm{~Hz}), 0.98(\mathrm{~s}, 9 \mathrm{H}), 0.25(\mathrm{~s}, 6 \mathrm{H}) ;{ }^{13} \mathrm{C} \mathrm{NMR}\left(125 \mathrm{MHz}, \mathrm{CDCl}_{3}\right)$ $\delta 197.2$ (x2), 161.2, 148.2, 136.2, 129.4, 127.5, 125.9, 116.6, 100.3, 47.4, 46.3, 28.3, 25.4 (x3), 24.4, 17.9 (x6), 18.1, 12.5 (x3), -4.5, -4.4; FTIR (neat) 2964, 1690, 1591, 1288; LRMS (CI) calcd for $\left[\mathrm{C}_{29} \mathrm{H}_{46} \mathrm{O}_{4} \mathrm{Si}_{2}\right]\left([\mathrm{M}]^{-}\right)$: 514 ; found $514 ;[\alpha]_{\mathrm{D}}^{23}+15.4$ (c 1.0, $\mathrm{CHCl}_{3},>99 \%$ ee). Regioselectivity was determined by ${ }^{1} \mathrm{H}$ NMR analysis of the crude mixture: $\delta 3.43$ (dd, $1 \mathrm{H}, J=$ 12.0, $5.5 \mathrm{~Hz}$, major), $3.38(\mathrm{dd}, 1 \mathrm{H}, J=11.5,6.0 \mathrm{~Hz}$, minor), $3.25(\mathrm{dd}, 1 \mathrm{H}, J=12.0,6.0 \mathrm{~Hz}$, minor), 3.04 (dt, $1 \mathrm{H}, J=7.5,5.5 \mathrm{~Hz}$, major). Enantioselectivity was determined by HPLC analysis using a Daicel Chiralcel OD-H column $(0.2 \% i-\mathrm{PrOH}$ in hexanes for elution; $1 \mathrm{~mL} / \mathrm{min}$; $\lambda 254 \mathrm{~nm}$ ); retention times: $9.43 \mathrm{~min}$ (regioisomer), $10.19 \mathrm{~min}$ (major), $12.30 \mathrm{~min}$ (minor). The absolute configuration was determined by analogy with (+)-(4aS, 9aR)-1, 4, 4a, 9a-tetrahydro-2(triisopropylsilyl)oxy-anthracene-9, 10-dione. 